\title{
PRODUÇÃO DE FRUTOS E CARACTERÍSTICAS MORFOFISIOLÓGICAS DE Schinus terebinthifolius Raddi., NA REGIÃO DO BAIXO SÃO FRANCISCO, BRASIL ${ }^{1}$
}

Danilla Cristina Lemos Souza², Renata Silva-Mann³, Robério Anastácio Ferreira ${ }^{3}$, Laura Jane Gomes ${ }^{3}$, Thalyta dos Santos Almeida ${ }^{4}$, Andreza dos Santos Oliveira ${ }^{4}$, Glauber Santos Pereira ${ }^{4}$ e Itamara Bomfim Gois ${ }^{5}$

RESUMO - Espécies florestais com potencial econômico, como é o caso da aroeira (Schinus terebinthifolius Raddi.), requerem a seleção de indivíduos com características superiores para uso em projetos de restauração florestal e para o estabelecimento de plantios comerciais. Estas plantações podem contribuir para a sustentabilidade de populações naturais de espécies nos fragmentos florestais remanescentes, em áreas de preservação permanente, reservas legais ou outras áreas de importância ecológica. Foi avaliada a produção de frutos, morfometria e viabilidade de sementes de 15 indivíduos de aroeira, em diferentes fragmentos, na região do Baixo São Francisco sergipano. A produção de frutos foi estimada pelo índice de intensidade de Fournier e as características morfométricas foram obtidas com paquímetro e balança analítica digital. A viabilidade e o vigor foram avaliados por meio da porcentagem de germinação e índice de velocidade de germinação (IVG), sob condições controladas. Os resultados da produção de frutos foram submetidos à análise de variância e as médias comparadas pelo teste de Tukey $(p<0,05)$. Para as demais variáveis foi utilizado o teste de Scott-Knott $(p<0,05)$. Os indivíduos diferiram em índices de Fournier (índices 1, 2, 3 e 4) e no tamanho de frutos e sementes. A germinação variou de 0 a $83 \%$ e o IVG de 0,00 a 0,98. As diferenças fenotípicas observadas entre os indivíduos para a produção de frutos e características morfofisiológicas podem ser exploradas em restauração florestal e estabelecimento de testes de procedências/progênies, visando à discriminação de material superior para futuros plantios comerciais.

Palavras-chave: Aroeira; Morfometria; Viabilidade de sementes.

\section{FRUIT YIELD AND MORPHOPHYSIOLOGICAL CHARACTERISTICS OF Schinus terebinthifolius Raddi., IN LOWER REGION OF SAN FRANCISCO, BRAZIL}

\begin{abstract}
Forest species with economic potential, such as aroeira (Schinus terebinthifolius Raddi.), require selection of individuals with superior characteristics for use in forest restoration projects and for establishment of commercial plantations. These plantations can contribute to the sustainability of natural populations of species in the remaining forest fragments, in areas of permanent preservation, legal reserves or other areas of ecological significance. It was evaluated the fruits' yield, morphometry and viability of seeds from 15 individuals of aroeira, collected in different fragments in the region of Lower São Francisco river, Sergipe State. The fruit yield was estimated by Fornier's intensity index, and the morphometric characteristics were obtained with a digital paquimeter and analytical balance. The viability and vigor were evaluated by germination percentage and germination speed index (GSI), under controlled conditions. The results of fruit yield were subjected to variance analysis, and the means were compared by Tukey $(p<0.05)$. The other variables means
\end{abstract}

\footnotetext{
${ }^{1}$ Recebido em 09.05.2012 aceito para publicação em 13.08.2013.

${ }^{2}$ Programa de Pós-Graduação em Ciência Florestal da Universidade Estadual Paulista Júlio de Mesquita Filho, UNESP, Brasil. E-mail: <danillacristina@ig.com.br>.

3Centro de Ciências Agrárias da Universidade Federal de Sergipe, UFS, Brasil.E-mail: <renatamann@hotmail.com>e <raf@infonet.com.br> e<laurabuturi@gmail.com>.

${ }^{4}$ Programa de Pós-Graduação em Agroecossistemas da Universidade Federal de Sergipe, UFS, Brasil. E-mail: <thalyta_sa@hotmail.com>, <andrezas_oliveiras@hotmail> e <glaubinhose@hotmail.com>.

${ }^{5}$ Programa de Pós-Graduação em Fitotecnia da Universidade Federal de Viçosa, UFV, Brasil. E-mail: <itamarafloresta@gmail.com>.
} 
were compared by Scott-Knott test ( $p<0.05)$. The individuals differed in Fournier's indices (indices 1, 2, 3 and 4) and in the size of fruits and seeds. The germination varied from 0 to $83 \%$ and the GSI from 0.00 to 0.98. The phenotypic differences observed among individuals for fruit yield, and morphophysiological characteristics can be used in forest restoration and establishment of provenances/progenies tests, aiming discrimination of superior material for future commercial plantations.

Keywords: Aroeira; Morphometry; Seeds viability.

\section{INTRODUÇÃO}

A ausência de variação genética em sementes florestais utilizadas para produção de mudas nos projetos de recuperação de áreas degradadas, decorrente da seleção de árvores-matriz geneticamente semelhantes entre si, afeta a sustentabilidade das comunidades vegetais recuperadas. Para que uma espécie seja efetivamente representada em programas de recuperação florestal e melhoramento, e não apresente problemas futuros de frutificação ou de estabelecimento de seus propágulos, os indivíduos utilizados como árvoresmatriz devem possuir um conjunto de genes representativos daquela espécie ou população local, de modo a evitar descendentes pouco vigorosos e com baixo potencial de adaptação (RODRIGUES et al., 2009).

Assim, na atividade de colheita de sementes, o ideal é que se procure reunir sempre descendentes de um maior número possível de plantas genitoras. Considerando-se um número mínimo de indivíduos para um dado local, sugere-se a conservação genética em curto prazo (10 gerações da espécie), minimizando os danos por depressão endogâmica, sendo necessário um tamanho efetivo da população ( $\mathrm{Ne}$ ) de 50 , que equivale à colheita de sementes em 12 árvores-matriz, desde que elas ou os pais não sejam aparentados. Esse parâmetro $(\mathrm{Ne})$ representa o tamanho da amostra que garante a representatividade genética de uma população coletada em relação à população parental (VENCOVSKY, 1987). No entanto, com a redução das áreas de florestas naturais e o isolamento das florestas remanescentes, promovidos pela ação antrópica, há um decréscimo no número de indivíduos de populações naturais, dificultando, assim, a seleção de indivíduos superiores.

A estimativa da intensidade da produção de frutos por indivíduo e por região pode ser importante para a coleta de sementes visando à produção de mudas para recuperação de áreas degradadas, à conservação ex situ e à exploração do germoplasma (VIEIRA; CARVALHO, 2009). Associado a isso, estudos de produção de frutos em diferentes anos e períodos ao longo dos anos permitem obter uma estimativa mais precisa da produção de frutos, uma vez que a taxa de frutificação pode variar entre populações, entre indivíduos e entre anos (STEPHENSON, 1981). Além disso, permitem avaliar também a influência dos fatores climáticos (sazonalidade) e da exploração antrópica sobre a intensidade da produção.

Na região do Baixo São Francisco sergipano, o decréscimo das populações naturais tem sido observado para aroeira (Schinus terebinthifolius Raddi.) que, dentre as espécies utilizadas em projetos de recuperação florestal, destaca-se por ser uma espécie nativa de crescimento rápido e comportamento típico de espécies pioneiras. A intensa exploração da aroeira nessa região, para uso como pimenta-rosa nas indústrias de alimentos, bem como de seus óleos essenciais em fármacos e cosméticos, implica na necessidade de obtenção de sementes com boa qualidade física e fisiológica para produção de mudas. Nessas condições, para a colheita de sementes, critérios devem ser adotados com o intuito de melhor utilizar a variabilidade genética disponível nas populações naturais remanescentes e evitar sementes provenientes de cruzamentos entre indivíduos relacionados por parentesco.

Para a manutenção da variabilidade genética atual nessas áreas, medidas conservativas devem ser tomadas. Em espécies florestais de valor econômico, o estabelecimento de plantios comerciais pode ser visto como uma alternativa para a conservação da espécie, uma vez que diminuirá as pressões nessas áreas, contribuindo, assim, para a sustentabilidade das populações. Nesse sentido, torna-se importante a seleção de indivíduos com caracteres superiores para tal finalidade.

Visando à indicação de indivíduos para produção comercial e elaboração de estratégias para conservação da aroeira na região do Baixo São Francisco sergipano, objetivou-se com o presente estudo avaliar a produção de frutos e as características 
morfofisiológicas de frutos e sementes de indivíduos remanescentes dessa região.

\section{MATERIAL E MÉTODOS}

O estudo foi realizado em fragmentos de mata ciliar de diferentes regiões fitogeográficas do Estado de Sergipe, situados na região do Baixo Curso do Rio São Francisco. O primeiro fragmento está localizado no município de Brejo Grande (10²8’40,2"S; 36²8’33,0"W), que possui clima do tipo megatérmico seco a sub-úmido, com temperatura média anual de $26^{\circ} \mathrm{C}$ e precipitação pluviométrica média anual de $1.200 \mathrm{~mm}$. O segundo fragmento está localizado no município de Neópolis (10¹8’29,8"S; 36³5’05,1"W), que apresenta condições climáticas semelhantes ao anterior e período chuvoso de março a agosto. O último fragmento está localizado no município de Propriá (10¹2’40,0"S; 3650’25,0"W), que apresenta clima megatérmico Semiárido, temperatura média no ano de $28^{\circ} \mathrm{C}$, precipitação pluviométrica média anual de $800 \mathrm{~mm}$ e período chuvoso de março a agosto (SERGIPE. SEPLANTEC/SUPES, 2000).

Cinco indivíduos de cada fragmento foram selecionados levando-se em consideração os que apresentaram bom aspecto fitossanitário (ausência de rachaduras no tronco e galhos cortados e ausência de pragas e patógenos) e evidência de frutificação. O número reduzido de indivíduos estudados deve-se principalmente, ao processo de ocupação humana que ocorreu nas vegetações ciliares do Estado de Sergipe, promovendo o isolamento dos fragmentos e possível perda de vigor dos indivíduos, uma vez que não foi observada frutificação para a maioria deles.

As observações fenológicas se deram no período de abril a junho de 2011. A produção de frutos de cada indivíduo de aroeira foi incluída em uma das cinco categorias de intensidade de Fournier (1974), onde: 0 - ausência da fenofase; 1 - 1\% a 25 \% da copa apresentando a fenofase; $2-26 \%$ a $50 \%$ da copa apresentando a fenofase; $3-51 \%$ a $75 \%$ da copa apresentando a fenofase; e $4-76 \%$ a $100 \%$ da copa apresentando a fenofase. Indivíduos nas categorias de intensidade 1 e 2 foram classificados como de baixa produção e indivíduos nas categorias acima de 2 foram considerados como de alta produção (PAISE; VIEIRA, 2005).

As colheitas foram realizadas em junho, sendo padronizada a retirada de $80 \%$ dos frutos presentes em cada indivíduo. Os restantes 20 \% foram mantidos nas árvores para suprir o banco de sementes do solo. Os frutos foram colhidos diretamente nas árvores, acondicionados em sacos de polietileno e levados ao Laboratório de Tecnologia de Sementes, no Departamento de Engenharia Agronômica, na Universidade Federal de Sergipe. Os mesmos foram colocados para secar a sombra por oito dias, e, em seguida, foram beneficiados, embalados em sacos plásticos transparentes impermeáveis, pesados e armazenados em câmara fria (6-8 ${ }^{\circ} \mathrm{C}$ e $60-65 \%$ de umidade) por 60 dias. Cada indivíduo constituiu um lote de sementes e a identidade dos mesmos foi mantida durante todo o período experimental.

Para uma estimativa da produção total de frutos de cada fragmento, foram somados os valores atribuídos a cada indivíduo $(\mathrm{kg})$, situados em um mesmo fragmento (PAISE; VIEIRA, 2005).

A descrição morfométrica foi realizada utilizandose, aleatoriamente, 400 frutos e 400 sementes de cada lote (quatro repetições de 100 frutos e 100 sementes de cada indivíduo) para medições de comprimento, largura e espessura, todas obtidas com paquímetro digital. Para obtenção da massa fresca de sementes, foram utilizadas oito amostras de 100 sementes por lote, pesadas em balança de precisão analítica.

O teor inicial de água de cada lote de sementes foi determinado conforme prescrições das Regras para Análise de Sementes (BRASIL, 2009), utilizando-se quatro repetições de 25 sementes.

A determinação da viabilidade das sementes foi realizada por meio de teste de germinação, com quatro repetições de 25 sementes para cada lote, semeadas em gerbox sobre o substrato areia e mantidas em câmara de germinação tipo BOD $\left(25^{\circ} \mathrm{C}\right.$, luz branca contínua por 30 dias). As avaliações foram realizadas a cada dois dias, considerando-se germinadas as plântulas com todas as estruturas desenvolvidas (raiz primária, hipocótilo, cotilédones, epicótilo e protófilos abertos), conforme sugerido pelas Regras para Análise de Sementes (BRASIL, 2009). Ao final do teste, as sementes foram avaliadas por meio dos parâmetros porcentagem de germinação e índice de velocidade de germinação (IVG), empregando-se as fórmulas propostas por Maguire (1962).

As sementes não germinadas foram avaliadas para determinação das sementes duras, dormentes e deterioradas, como recomendado pelas Regras para Análise de Sementes (BRASIL, 2009). 
Os dados de produção de frutos foram submetidos à análise de variância e as médias comparadas pelo teste de Tukey $(\mathrm{p}<0,05)$, por meio do programa estatístico SISVAR (FERREIRA, 2008). Para as demais variáveis foi utilizado o teste de Scott-Knott $(\mathrm{p}<0,05)$.

\section{RESULTADOS}

Para nove indivíduos de aroeira observou-se alta produção de frutos (Tabela 1).

Com exceção dos indivíduos P1 e N1, foi obtido um índice comum aos indivíduos pertencentes ao fragmento situado no município de Propriá e aos pertencentes ao fragmento situado no município de Neópolis, sendo estes os índices 2 e 3, respectivamente. Porém, o mesmo não ocorreu com os indivíduos de Brejo Grande, que ficaram distribuídos nos diferentes índices de Fournier (índices 1, 3 e 4).
Paralelamente, ao mensurar a biomassa de frutos produzida por indivíduo, resultados semelhantes foram encontrados, em analogia com o índice de Fournier, de modo que os indivíduos com maiores e menores intensidade de produção de frutos foram os que apresentaram maiores e menores biomassas, respectivamente. O valor médio obtido para esta variável foi de $2,170 \mathrm{~kg}$ e não houve distinção estatística significativa entre as biomassas de frutos produzidas por fragmento, sendo que o de Neópolis foi o mais produtivo e o de Propriá o menos produtivo.

As características morfométricas de frutos e sementes dos indivíduos de aroeira são apresentadas na Tabela 2.

Não houve um padrão na classificação dos indivíduos. Para todas as características estudadas, houve diferença significativa entre os indivíduos de

Tabela 1 - Estimativas do índice de intensidade de Fournier para fenofase de frutificação (0 - ausência da fenofase; 1 $1 \%$ a $25 \%$ da copa apresentando a fenofase; $2-26 \%$ a $50 \%$ da copa apresentando a fenofase; $3-51 \%$ a $75 \%$ da copa apresentando a fenofase; e $4-76 \%$ a $100 \%$ da copa apresentando a fenofase) e da produção de frutos (baixa produção - $\leq 50 \%$ da copa apresentando a fenofase; alta produção - > 50\% da copa apresentando a fenofase) de indivíduos de aroeira (Schinus terebinthifolius Raddi.), biomassa de frutos produzida por indivíduo e estimativa da biomassa de frutos produzida por fragmento, em diferentes regiões no Baixo São Francisco sergipano (BG - Brejo Grande; e N - Neópolis; e P - Propriá).

Table 1 - Estimate of the Fornier intensity index for fructification phenophase ( 0 -absence of frutification; 1 - $1 \%$ to $25 \%$ of the crown showing fruits ; $2-26 \%$ to $50 \%$ of the crown showing fruits the phenophase; $3-51 \%$ to $75 \%$ of the crown showing fruits; and $4-76 \%$ to $100 \%$ of the crown showing fruits) and the fruits production (low production - $\leq 50 \%$ of the crown showing fruits; high production - $>50 \%$ of the crown showing fruits) of the individuals of aroeira (Schinus terebinthifolius Raddi.), fruits biomass produced by each individual and estimate of the fruits biomass produced by fragment, in different regions of Low São Francisco river, Sergipe State (BG-Brejo Grande; N-Neópolis; and P-Propriá).

\begin{tabular}{|c|c|c|c|c|c|c|c|c|c|}
\hline \multirow{2}{*}{$\begin{array}{l}\text { Indivíduo/ } \\
\text { lote }\end{array}$} & \multicolumn{5}{|c|}{ Índice de Intensidade de Fournier } & \multicolumn{2}{|c|}{$\begin{array}{l}\text { Produção } \\
\text { de frutos }\end{array}$} & \multirow{2}{*}{$\begin{array}{l}\text { Biomassa de } \\
\text { frutos(Kg) }\end{array}$} & \multirow{2}{*}{$\begin{array}{l}\text { Biomassa de frutos } \\
\text { por fragmento }(\mathrm{Kg})\end{array}$} \\
\hline & 0 & 1 & 2 & 3 & 4 & Baixa & Alta & & \\
\hline BG1 & & & & & $\mathrm{X}$ & & $\mathrm{X}$ & 3,563 & \\
\hline BG2 & & $\mathrm{X}$ & & & & $\mathrm{X}$ & & 0,725 & \\
\hline BG3 & & $\mathrm{X}$ & & & & $X$ & & 0,855 & 10,581 a \\
\hline BG4 & & & & $X$ & & & $\mathrm{X}$ & 2,755 & \\
\hline BG5 & & & & $\mathrm{X}$ & & & $\mathrm{X}$ & 2,683 & \\
\hline N1 & & & & & $\mathrm{X}$ & & $X$ & 3,192 & \\
\hline N2 & & & & $\mathrm{X}$ & & & $\mathrm{X}$ & 2,194 & \\
\hline N3 & & & & $\mathrm{X}$ & & & $\mathrm{X}$ & 2,044 & 12,192 a \\
\hline N4 & & & & $X$ & & & $\mathrm{X}$ & 2,031 & \\
\hline N5 & & & & $X$ & & & $X$ & 2,731 & \\
\hline P 1 & & & & $\mathrm{X}$ & & & $\mathrm{X}$ & 2,587 & \\
\hline P 2 & & & $X$ & & & $X$ & & 1,981 & \\
\hline P 3 & & & $\mathrm{X}$ & & & $X$ & & 1,413 & 9,778 а \\
\hline P 4 & & & $X$ & & & $\mathrm{X}$ & & 1,817 & \\
\hline P 5 & & & $X$ & & & $\mathrm{X}$ & & 1,980 & \\
\hline
\end{tabular}

Valores seguidos pela mesma letra, nas colunas, não diferem entre si pelo teste de Tukey a 5\% de probabilidade.

Revista Árvore, Viçosa-MG, v.37, n.5, p.923-932, 2013 
Tabela 2 - Comparação das médias das características morfométricas (Comp F - comprimento do fruto; Larg F - largura do fruto; Espes F - espessura do fruto; Comp S - comprimento da semente; Larg S - largura da semente; Espes $\mathrm{S}$ - espessura da semente; MF S - massa fresca de 1.000 sementes) de indivíduos de aroeira (Schinus terebinthifolius Raddi.) procedentes de diferentes fragmentos no Baixo São Francisco sergipano (BG - Brejo Grande; N - Neópolis; e P - Propriá).

Table 2 - Mean comparison for morphometric characteristics (Comp F - fruit length; Larg F - fruit width; Espes F-fruit thickness; Comp S - seed length; Larg S - seed width; Espes S - seed thickness; MF S - fresh mass of 1,000 seeds) of aroeira individuals (Schinus terebinthifolius Raddi.) obtained from different fragments of low São Francisco river, Sergipe State (BG - Brejo Grande; N-Neópolis; and P-Propriá).

\begin{tabular}{|c|c|c|c|c|c|c|c|}
\hline Indivíduo & Comp F & Larg F & Espes F & Comp S & Larg S & Espes S & MF S \\
\hline BG1 & $4.47 \mathrm{~d}$ & $4.13 \mathrm{c}$ & $3.89 \mathrm{c}$ & $3.70 \mathrm{f}$ & $3.15 \mathrm{~d}$ & $1.91 \mathrm{~d}$ & $1.47 \mathrm{e}$ \\
\hline BG2 & $4.77 \mathrm{a}$ & $4.27 \mathrm{~b}$ & $3.98 \mathrm{~b}$ & $4.02 \mathrm{a}$ & $3.17 \mathrm{~d}$ & $2.02 \mathrm{~b}$ & $1.82 \mathrm{~b}$ \\
\hline BG3 & $4.51 \mathrm{c}$ & $4.14 \mathrm{c}$ & $3.81 \mathrm{~d}$ & $3.62 \mathrm{~h}$ & $3.14 \mathrm{~d}$ & $1.93 \mathrm{~d}$ & $1.60 \mathrm{~d}$ \\
\hline BG4 & $4.69 \mathrm{~b}$ & $4.31 \mathrm{a}$ & $3.98 \mathrm{~b}$ & 3.92 b & $3.23 \mathrm{c}$ & $1.88 \mathrm{~d}$ & $1.96 \mathrm{a}$ \\
\hline BG5 & $4.43 \mathrm{~d}$ & $4.13 \mathrm{c}$ & $3.74 \mathrm{e}$ & $3.64 \mathrm{~g}$ & $3.16 \mathrm{~d}$ & $1.84 \mathrm{e}$ & $1.41 \mathrm{e}$ \\
\hline N1 & $4.14 \mathrm{~g}$ & $4.17 \mathrm{c}$ & $3.56 \mathrm{f}$ & $3.51 \mathrm{i}$ & $3.09 \mathrm{e}$ & $1.80 \mathrm{f}$ & $1.56 \mathrm{~d}$ \\
\hline N2 & $4.32 \mathrm{e}$ & $4.22 \mathrm{~b}$ & 3.68 e & $3.71 \mathrm{f}$ & $3.28 \mathrm{~b}$ & $1.96 \mathrm{c}$ & $1.65 \mathrm{~d}$ \\
\hline N3 & $3.96 \mathrm{~h}$ & $3.82 \mathrm{f}$ & $3.43 \mathrm{~g}$ & $3.33 \mathrm{j}$ & $2.94 \mathrm{f}$ & $1.58 \mathrm{~g}$ & $1.28 \mathrm{f}$ \\
\hline N4 & $4.32 \mathrm{e}$ & $4.23 \mathrm{~b}$ & $3.68 \mathrm{e}$ & $3.81 \mathrm{~d}$ & $3.33 \mathrm{a}$ & $1.79 \mathrm{f}$ & $1.66 \mathrm{~d}$ \\
\hline N5 & $4.19 \mathrm{f}$ & $4.08 \mathrm{~d}$ & $3.53 \mathrm{f}$ & $3.87 \mathrm{c}$ & $3.37 \mathrm{a}$ & $1.90 \mathrm{~d}$ & $1.48 \mathrm{e}$ \\
\hline $\mathrm{P} 1$ & $4.75 \mathrm{a}$ & $4.33 \mathrm{a}$ & $4.06 \mathrm{a}$ & $3.74 \mathrm{e}$ & $3.19 \mathrm{c}$ & $2.05 \mathrm{a}$ & $1.68 \mathrm{c}$ \\
\hline P 2 & $4.32 \mathrm{e}$ & $3.88 \mathrm{e}$ & $3.37 \mathrm{~h}$ & $3.66 \mathrm{~g}$ & $3.04 \mathrm{e}$ & $1.97 \mathrm{c}$ & $1.47 \mathrm{e}$ \\
\hline P 3 & $4.41 \mathrm{~d}$ & $3.88 \mathrm{e}$ & $3.68 \mathrm{e}$ & $3.58 \mathrm{~h}$ & $2.93 \mathrm{f}$ & $1.98 \mathrm{c}$ & $1.63 \mathrm{~d}$ \\
\hline P 4 & $4.67 \mathrm{~b}$ & $4.05 \mathrm{~d}$ & $3.77 \mathrm{~d}$ & $3.82 \mathrm{~d}$ & $2.96 \mathrm{f}$ & $2.07 \mathrm{a}$ & $1.73 \mathrm{c}$ \\
\hline P 5 & $4.43 \mathrm{~d}$ & $3.92 \mathrm{e}$ & $3.70 \mathrm{e}$ & $3.58 \mathrm{~h}$ & $2.89 \mathrm{~g}$ & $1.93 \mathrm{~d}$ & $1.70 \mathrm{c}$ \\
\hline
\end{tabular}

Médias seguidas pela mesma letra, nas colunas, não diferem entre si pelo teste de Scott-Knott a 5\% de probabilidade.

aroeira. Observou-se amplitude de 3,96 a 4,77 mm em comprimento, 3,82 a 4,33 mm em largura e 3,37 a 4,05 mm em espessura para os frutos. Nos indivíduos BG2, BG4 e P1 observou-se valores superiores para todas as características de frutos, enquanto que para o indivíduo N3 registrou-se os menores valores. Verificou-se maior comprimento nas sementes dos indivíduos BG2 e BG4 (4,02 mm e 3,92 $\mathrm{mm}$, respectivamente) e menor nos indivíduos N3 e N1 (3,33 mm e 3,51 mm, respectivamente); maior largura nos indivíduos N5, N4 e N2 (3,37 mm, $3,33 \mathrm{~mm}$ e 3,28 $\mathrm{mm}$, respectivamente) e menor nos indivíduos P5, P3, N3 e P4 (2,89 mm, 2,93 mm, 2,94 mm e 2,96 mm, respectivamente); e para espessura destacaram-se os indivíduos P4, P1, e BG2 (2,07 mm, 2,05 mm e 2,02 mm, respectivamente) com maiores valores, e os indivíduos N3, N4 e N1 (1,58 mm, 1,79 mm e 1,80 mm, respectivamente) com menores valores. Em relação à massa fresca das sementes, maiores valores foram observados para os indivíduos BG4 e BG2 (1,96 mm e 1,82 mm, respectivamente) e menores para os indivíduos N3, BG5, BG1, P2 e N5 (1,28 mm, 1,41 mm, 1,47 mm, 1,47 mm e 1,48 mm, respectivamente). É interessante observar que o indivíduo N3 apresentou valores inferiores para todas as características morfométricas avaliadas e que não houve médias comuns para frutos e sementes de indivíduos pertencentes a um mesmo fragmento.

O teor de água inicial das sementes dos diferentes indivíduos variou de 11,59\% (P3) a 33,26\% (BG5) (Tabela 3). Sementes com teor de água acima de $20 \%$ apresentaram baixa porcentagem de germinação (0-18\%).

Houve diferença significativa entre os indivíduos de aroeira estudados, com exceção do parâmetro plântulas anormais (Tabela 3). A germinação variou entre germinação nula (BG4) a 83 \% (N1), sendo que os indivíduos BG1, N1, N2, P2 e P3 apresentaram germinação superior a 50 \%.

Algumas sementes que emitiram radícula não conseguiram, até o final do teste, estabelecer plântulas. O percentual de plântulas normais foi maior que o de plântulas anormais. O percentual de sementes deterioradas foi baixo (0-14\%) para todos os indivíduos.

As sementes do indivíduo N1 apresentaram maior IVG, necessitando de um tempo menor de incubação e mostrando-se mais vigorosas.

Ao comparar os dados de massa fresca e percentual de germinação das sementes estudadas nota-se que não houve relação positiva entre eles (Figura 1). 
Tabela 3 - Teor de água (U) e comparação de médias para porcentagem de emissão de radícula (ER), de germinação (Germ.), de sementes deterioradas (Deter.), dormentes (Dorm.) e duras (Dur.), porcentagem de plântulas normais (N) e anormais (A) e índice de velocidade de germinação (IVG) de sementes de aroeira (Schinus terebinthifolius Raddi.) oriundas de diferentes regiões no Baixo São Francisco sergipano (BG - Brejo Grande; N - Neópolis; e P - Propriá).

Table 3 - Water content (U) and mean comparison for radicle emission percentage (ER), germination (Germ.), deteriorate seeds (Deter.), dormant seeds (Dorm.), and hardseedness (Dur.), percentage of normal seedlings ( $N$ ), and abnormal seedlings (A), and Speed Germination Index (IVG) of aroeira (Schinus terebinthifolius Raddi.) seeds, obtained from different regions of Low São Francisco river, Sergipe State (BG - Brejo Grande; and N-Neópolis; and P-Propriá).

\begin{tabular}{|c|c|c|c|c|c|c|c|c|c|}
\hline \multirow{2}{*}{ Indivíduo } & \multicolumn{6}{|c|}{ Sementes (\%) } & \multicolumn{2}{|c|}{ Plântulas (\%) } & \multirow{2}{*}{ IVG } \\
\hline & $\mathrm{U}$ & ER & Germ. & Deter. & Dorm. & Dur. & $\mathrm{N}$ & A & \\
\hline BG1 & 15,50 & 70,0 a & 56,0 a & $0,0 \mathrm{~b}$ & $30,0 \mathrm{c}$ & $0,0 \mathrm{~b}$ & 56,0 a & 0,0 a & $0.62 \mathrm{~b}$ \\
\hline BG2 & 15,91 & $16,0 \mathrm{c}$ & $16,0 \mathrm{~b}$ & $2,0 \mathrm{~b}$ & 82,0 a & $0,0 \mathrm{~b}$ & $16,0 \mathrm{~b}$ & 0,0 a & $0.17 \mathrm{c}$ \\
\hline BG3 & 26,62 & $18,0 \mathrm{c}$ & $16,0 \mathrm{~b}$ & $0,0 \mathrm{~b}$ & 82,0 a & $0,0 \mathrm{~b}$ & $16,0 \mathrm{~b}$ & 0,0 a & $0.19 \mathrm{c}$ \\
\hline BG4 & 21,30 & $0,0 \mathrm{~d}$ & 0,0 с & $4,0 \mathrm{~b}$ & 0,0 e & 96,0 a & $0,0 \mathrm{c}$ & 0,0 a & $0.00 \mathrm{c}$ \\
\hline BG5 & 33,26 & $18,0 \mathrm{c}$ & $18,0 \mathrm{~b}$ & $2,0 \mathrm{~b}$ & 80,0 a & $0,0 \mathrm{~b}$ & $16,0 \mathrm{~b}$ & $2,0 \mathrm{a}$ & $0.21 \mathrm{c}$ \\
\hline N1 & 15,19 & 83,0 a & 83,0 a & $0,0 \mathrm{~b}$ & $17,0 \mathrm{~d}$ & $0,0 \mathrm{~b}$ & 78,0 a & 5,0 a & 0.98 a \\
\hline N2 & 12,68 & $56,0 \mathrm{~b}$ & 56,0 a & $2,0 \mathrm{~b}$ & $39,0 \mathrm{c}$ & $3,0 \mathrm{~b}$ & 53,0 a & 3,0 a & $0.59 \mathrm{~b}$ \\
\hline N3 & 13,83 & 27,0 c & $21,0 \mathrm{~b}$ & 14,0 a & $59,0 \mathrm{~b}$ & $0,0 \mathrm{~b}$ & $19,0 \mathrm{~b}$ & $2,0 \mathrm{a}$ & $0.25 \mathrm{c}$ \\
\hline N4 & 24,16 & $4,0 \mathrm{~d}$ & $4,0 \mathrm{c}$ & 11,0 a & 85,0 a & $0,0 \mathrm{~b}$ & $4,0 \mathrm{c}$ & 0,0 a & 0.05 c \\
\hline N5 & 14,88 & $15,0 \mathrm{c}$ & $14,0 \mathrm{~b}$ & $11,0 \mathrm{a}$ & 73,0 a & $1,0 \mathrm{~b}$ & $11,0 \mathrm{~b}$ & 3,0 a & $0.11 \mathrm{c}$ \\
\hline $\mathrm{P} 1$ & 15,51 & $48,0 \mathrm{~b}$ & 45,0 a & $2,0 \mathrm{~b}$ & $50,0 \mathrm{~b}$ & $0,0 \mathrm{~b}$ & 45,0 a & 0,0 a & $0.57 b$ \\
\hline P 2 & 15,01 & 65,0 a & 57,0 a & $4,0 \mathrm{~b}$ & $31,0 \mathrm{c}$ & $0,0 \mathrm{~b}$ & 56,0 a & $1,0 \mathrm{a}$ & $0.71 \mathrm{~b}$ \\
\hline P 3 & 11,59 & 70,0 a & 64,0 a & $2,0 \mathrm{~b}$ & 28,0 c & $0,0 \mathrm{~b}$ & 62,0 a & $2,0 \mathrm{a}$ & $0.75 \mathrm{~b}$ \\
\hline P 4 & 14,96 & $45,0 \mathrm{~b}$ & 45,0 a & $5,0 \mathrm{~b}$ & $50,0 \mathrm{~b}$ & $0,0 \mathrm{~b}$ & 45,0 a & 0,0 a & $0.41 \mathrm{c}$ \\
\hline P 5 & 12,80 & $47,0 \mathrm{~b}$ & 43,0 a & $1,0 \mathrm{~b}$ & $52,0 \mathrm{~b}$ & $0,0 \mathrm{~b}$ & 42,0 a & $1,0 \mathrm{a}$ & $0.48 \mathrm{~b}$ \\
\hline
\end{tabular}

Médias seguidas pela mesma letra, nas colunas, não diferem entre si pelo teste de Scott-Knott a 5\% de probabilidade.

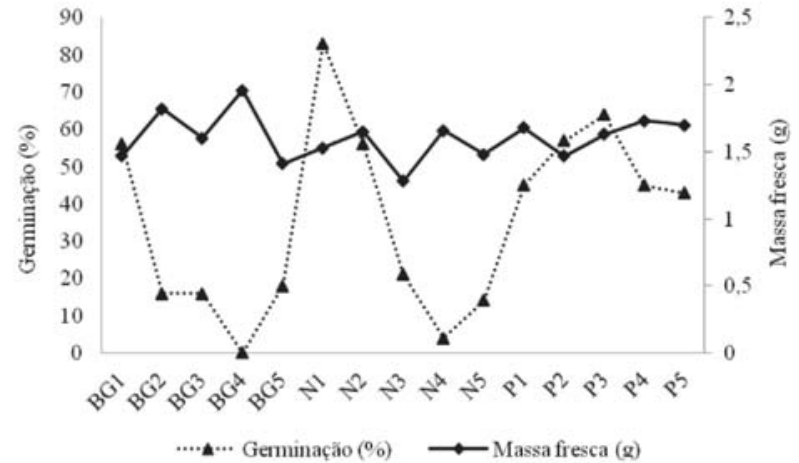

Figura 1 - Relação entre o percentual de germinação e a massa fresca de sementes de indivíduos de aroeira (Schinus terebinthifolius Raddi.), situados em diferentes fragmentos na região do Baixo São Francisco sergipano (BG - Brejo Grande, N Neópolis, P - Propriá).

Figure 1 - Relation between the germination percentage and the fresh mass of seeds from individuals of aroeira (Schinus terebinthifolius Raddi.), located in different fragments of Low Sergipean region of São Francisco river (BG-Brejo Grande, $N$-Neópolis, $P$-Propriá).
O indivíduo N1 não mostrou superioridade para a variável massa fresca de sementes, bem como para os demais parâmetros morfométricos avaliados. No entanto, foi o que apresentou melhores resultados para germinação e vigor, enquanto que o contrário foi observado para o indivíduo BG2.

Por meio dos dados de germinação, não se pode considerar que a germinação das sementes de aroeira foi determinada pela procedência das mesmas.

\section{DISCUSSÃO}

Os valores semelhantes de produção de frutos de indivíduos em um mesmo fragmento, bem como a diferença na categorização entre os fragmentos, podem estar associados ao fato dos indivíduos estarem sujeitos às mesmas condições edafoclimáticas, onde o fator ambiente exerce influência sobre tal característica (MORELLATOetal., 2000). Newstrom et al. (1994) afirmam que, se avaliados em diferentes ecossistemas, os padrões fenológicos podem variar dentro de uma mesma espécie. No entanto, a ausência de uniformidade na produção 
de frutos para a classificação comum dos indivíduos situados no fragmento de Brejo Grande sugere que os padrões de frutificação de aroeira são mais afetados pelas características intrínsecas aos indivíduos, uma vez que as informações genéticas contidas em cada indivíduo são determinantes nos eventos fenológicos (ALENCAR, 1994).

Segundo coletores locais de frutos de aroeira da região estudada, uma árvore chega a produzir cerca de 4-6 kg de frutos ao ano, o que permite inferir que a maioria dos indivíduos produziu elevada biomassa de frutos, visto que tais valores foram obtidos em apenas uma coleta. Por outro lado, apesar da intensa frutificação observada na maioria dos indivíduos coletados, evidenciou-se um número relativamente reduzido de indivíduos em evento de frutificação em cada área, o que reforça a necessidade de manutenção de matrizes em planos de manejo, sob pena de acentuar os efeitos de endogamia nos processos de exploração (MANTOVANI et al., 2003).

Observou-se uma menor produção de frutos no fragmento Propriá, que pode estar diretamente associada às condições ambientais da área, que, diferente das outras, apresenta clima Semiárido. A disponibilidade de água durante o florescimento representa fator relevante na produtividade da população. Nessa fase, o principal efeito do déficit hídrico é a redução do número de sementes, uma vez que a menor disponibilidade de água promove decréscimos da fotossíntese, com prejuízos à produção (MARCOS FILHO, 2005).

As análises morfométricas são importantes para verificação das interações genótipo e ambiente, uma vez que ocorrem variações fenotípicas em indivíduos de uma mesma espécie (CARDOSO; LOMÔNACO, 2003). Frutos e sementes de espécies nativas apresentam desuniformidade nos aspectos reprodutivos, e precisam ser estudados para que sejam estabelecidos critérios de seleção como cor, tamanho, espessura, entre outros (BORGES et al., 2010).

A variação obtida para os frutos e sementes de aroeira aqui estudada pode ocorrer em função das características genéticas de cada indivíduo. Soma-se a isso a segregação causada pela alogamia, comum nas frutíferas (CARVALHO et al., 2003). Assim, a existência de variabilidade revela o potencial genético da espécie para a conservação de germoplasma e para a colheita de sementes (GUSMÃO et al., 2006), bem como a possibilidade de seleção de materiais promissores (MELCHIOR et al., 2006; MARIOT et al., 2009).

Ao propor padrões de germinação e teor de água para sementes de aroeira, Wielewicki et al. (2006) determinaram 48 \% de germinação para o teor máximo de água de $12,7 \%$ nas sementes. No presente estudo, sementes com baixo e alto teores de água apresentaram diferentes porcentagens de germinação.

Variações significativas na viabilidade de sementes de aroeira são esperadas devido à não domesticação da espécie e à origem do material experimental. Sementes de espécies silvestres, em seu estado natural, geralmente, comportam grande variabilidade genética, podendo resultar em variações nas características morfofisiológicas das sementes, que, por sua vez, podem influenciar no comportamento ecológico dos indivíduos de mesma espécie. Além disso, devido à ampla distribuição geográfica, essas espécies ficam sujeitas a variações edafoclimáticas em escalas espaciais e temporais (WIELEWICKI et al., 2006).

A germinação nula do indivíduo BG4 foi decorrente do elevado percentual de sementes duras (96\%), e a baixa germinação (4-21 \%) dos demais indivíduos provavelmente está relacionada à presença de dormência nas sementes, que, conforme Brasil (2009), sementes dormentes são aquelas que, durante o teste de germinação, absorvem água, mas não obtêm capacidade para germinar.

A aroeira é caracterizada por não apresentar dormência e ser classificada como ortodoxa (CARVALHO et al., 2006). No entanto, pode ter ocorrido a indução de dormência secundária nas sementes, devido às condições de armazenamento e à temperatura utilizada no teste de germinação. Segundo Bewley e Black (1994), a temperatura é um fator que influencia a germinação das sementes, determinando a capacidade e a velocidade de germinação, removendo a dormência primária e/ou secundária e induzindo a dormência secundária.

Ao estudar o papel de aves frugívoras na germinação de sementes da espécie, D’ávila et al. (2010) verificaram que sementes de aroeira ingeridas por determinadas espécies de aves germinaram antes que seus controles e apresentaram taxa de germinação mais alta que o controle, inferindo, assim, que os efeitos químico e mecânico desempenham papel no aumento da germinação das sementes. Nesse sentido, estudos que avaliem a dormência em aroeira são importantes para o conhecimento do comportamento ecológico da espécie. 
A ausência de capacidade das sementes que emitiram radícula em estabelecer plântulas pode estar relacionada ao baixo vigor, ou mesmo à baixa qualidade das sementes que pode ocorrer devido a inúmeros fatores, dentre eles problemas associados à ocorrência de fungos (de MARCHI et al., 2011). Por outro lado, o baixo percentual de sementes deterioradas comprova que, em sua maioria, as sementes estavam viáveis.

Além das porcentagens de germinação, que oferecem informações sobre as características de germinação do conjunto de sementes, o índice de velocidade de germinação permite interpretações sobre o vigor, indicando a demanda do tempo de incubação para que as sementes possam germinar, já que uma mesma espécie pode apresentar diferentes padrões de distribuição temporal da germinação(BORGHETTI; FERREIRA, 2004). As diferenças encontradas para este parâmetro podem ser explicadas pela heterogeneidade fisiológica apresentada por sementes de espécies florestais que, devido a diversos fatores, incluindo o período de colheita, condições de armazenamento e teor de água, afetam diretamente o vigor (PACHECO et al., 2011).

Ao comparar dados de massa fresca e percentual de germinação das sementes estudadas não houve relação positiva entre eles. O indivíduo N1 não mostrou superioridade para a variável massa fresca de sementes, bem como para os demais parâmetros morfométricos avaliados. No entanto, foi o que apresentou melhores resultados para germinação e vigor, enquanto que o contrário foi observado para o indivíduo BG2. Resultados similares foram encontrados por Ferreira e Torres (2000) ao estudar a influência do tamanho das sementes na germinação e no vigor de plântulas de Acacia senegal. Pereira et al. (2011) também verificaram que o tamanho de frutos e sementes não exerce influência sobre a germinação de sementes de jatobá-do-cerrado.

A relação positiva entre tamanho e porcentagem de germinação não se aplica genericamente a todas as espécies. Embora muitas vezes ocorra tal relação, as características físicas da semente não são determinantes para prever sua qualidade fisiológica (HANLEY et al., 2007; FARIA et al., 2009). De acordo com Malavasi e Malavasi (2001), a germinabilidade de sementes florestais não está condicionada ao tamanho da semente, mas sim aos fatores externos, como o ambiente em que se encontra, e também às características genéticas da mesma.

Diferente do observado por Alves et al., (2005) para sementes de Mimosa caesalpiniifolia Benth, e semelhante ao encontrado por Faria et al., (2009) para a espécie Brosimum gaudichaudii Tréc, não se pode considerar que os padrões de germinação das sementes de aroeira foram determinados pela procedência das mesmas, não esquecendo que no efeito procedência estão confundidos os fatores ambientais e genéticos. Somente os lotes de sementes oriundos do fragmento situado na região de Propriá apresentaram percentual de germinação uniforme, o que pode ser justificado pelos baixos percentuais e baixa variação $(11,59-15,51$ \%) no teor de água desses lotes.

\section{CONCLUSÃO}

As diferenças fenotípicas observadas entre indivíduos para produção de frutos e características morfofisiológicas, que podem ser provenientes tanto de variações genéticas quanto ambientais, podem ser aproveitadas para a restauração florestal e mediante o estabelecimento de testes de procedências/progênies discriminar material superior para futuros plantios comerciais.

\section{AGRADECIMENTOS}

À Capes, pela bolsa de mestrado concedida ao primeiro autor; e ao CNPq, por financiar o projeto "Estratégias para o manejo sustentável da aroeira (Schinus terebinthifolius Raddi.) no Baixo São Francisco - Sergipe/ Alagoas”, do qual este trabalho fez parte.

\section{REFERÊNCIAS}

ALENCAR, J. C. Fenologia de cinco espécies arbóreas tropicais de Sapotaceae, correlacionada a variáveis climáticas na Reserva Ducke, Manaus, AM. Acta Amazonica, v.24, n.4, p.161-182, 1994.

ALVES, E. U. et al. Influência do tamanho e da procedência de sementes Mimosa caesalpiniifolia Benth. sobre a germinação e vigor. Revista Árvore, v.29, n.6, p.877-885, 2005.

BEWLEY, J. D.; BLACK, M. Seeds: physiology of development and germination. New York: Plenum Press, 1994. 445p.

BORGES, K. C. F. et al. Rendimento de polpa e morfometria de frutos e sementes de pitangueirado-cerrado. Revista Brasileira de Fruticultura, v.32, n.2, p.471-478, 2010. 
BORGHETTI, F.; FERREIRA, A. G. Interpretação de resultados de germinação. In: FERREIRA, A. G.; BORGHETTI, F. (Ed.). Germinação: do básico ao aplicado. Porto Alegre: Artmed, 2004. p.209-222.

BRASIL. Ministério da Agricultura, Pecuária e Abastecimento. Secretaria de Defesa

Agropecuária. Regras para análise de sementes. Brasília: 2009. 399p.

CARDOSO, G. L.; LOMÔNACO, C. Variações fenotípicas e potencial plástico de Eugenia calycina Cambess. (Myrtaceae) em uma área de transição cerrado-vereda. Revista Brasileira de Botânica, v.26, n.1, p.131-140, 2003.

CARVALHO, J. E. U.; NAZARÉ, R. F. R.; NASCIMENTO, W. M. O. Características físicas e físico-químicas de um tipo de bacuri (Platonia insignis Mart.) com rendimento industrial superior. Revista Brasileira de Fruticultura, v.25, n.2, p.326-328, 2003.

CARVALHO, L. R.; SILVA, E. A. A.; DAVIDE, A. C. Classificação de sementes florestais quanto ao comportamento no armazenamento. Revista Brasileira de

Sementes, v.28, n.2, p.15-25, 2006.

CRUZ, E. D.; CARVALHO, J. E. U. Biometria de frutos e germinação de sementes de Couratari stellata A. C. Smith (Lecythidaceae), Acta Amazonica, v.33, n.3, p.381-388, 2003.

D'AVILA, G. et al. The role of avian frugivores on germination and potential seed dispersal of the Brazilian Pepper Schinus terebinthifolius. Biota Neotropica, v.10, n.3, p.45-51, 2010.

FARIA, R. A. P. G. et al. Características biométricas e emergência de plântulas de Brosimum gaudichaudii Tréc. oriundas de diferentes procedências do cerrado matogrossense. Revista Brasileira de Plantas Medicinais, v.11, n.4, p.414-421, 2009.

FERREIRA, D. F. SISVAR: um programa para análises e ensino de estatística. Revista Symposium, v.6, p.36-41, 2008.
FERREIRA, M. G. R.; TORRES, S. B. Influência do tamanho das sementes na germinação e no vigor de plântulas de Acacia senegal (L.) de Willd. Revista Brasileira de Sementes, v.22, n.1, p.271-275, 2000.

FOURNIER, L. A. Un metodo cuantitativo para la medición de características fenológicas en arboles. Turrialba, v.24, n.4, p.422-423, 1974.

GUSMÃO, E.; VIEIRA, F. A.; FONSECA JÚNIOR, E. M. Biometria de frutos e endocarpos de murici (Byrsonima verbascifolia Rich. ex A. Juss.). Cerne, v.12, n.1, p.84-91, 2006.

HANLEY, M. E. et al. Seed size and seedling growth: differential response of Australian and British Fabaceae to nutrient limitation. New Phytologist, v.174, n.2, p.381-388, 2007.

MAGUIRE, J. D. Speed of germination-aid selection evolution for sudling emergence and vigor. Crop Science, v.2, n.1, p.176-177, 1962.

MALAVASI, U. C.; MALAVASI, M. M. Influência do tamanho e do peso da semente na germinação e no estabelecimento de espécies de diferentes estágios da sucessão vegetal. Floresta e Ambiente, v.8, n.1, p.211-215, 2001.

MANTOVANI, M. et al. Fenologia reprodutiva de espécies arbóreas em uma formação secundária da floresta atlântica. Revista Árvore, v.27, n.4, p.451-458, 2003.

MARCHI, J. L.; CICERO, S. M.; GOMES JUNIOR, F. G. Utilização da análise computadorizada de plântulas na avaliação do potencial fisiológico de sementes de amendoim tratadas com fungicida e inseticidas. Revista Brasileira de Sementes, v.33, n.4, p.652-662, 2011.

MARCOS FILHO, J. Fisiologia de sementes de plantas cultivadas. Piracicaba: FEALQ, 2005. 465p.

MARIOT, M. P. et al. Variabilidade genética para caracteres morfológicos e fisiológicos em espinheira-santa (Maytenus ilicifolia (Schrad.) Planch. e $M$. aquifolium Mart.). Revista Brasileira de Plantas Medicinais, v.11, n.3, p.310-316, 2009.

Revista Árvore, Viçosa-MG, v.37, n.5, p.923-932, 2013 
MELCHIOR, S. J. et al. Colheita e armazenamento de sementes de gabiroba (Campomanesia adamantium Camb. - Myrtaceae) e implicações na germinação. Revista Brasileira de Sementes, v.28, n.3, p.141-150, 2006.

MORELLATO, L. P. C. et al. Phenology of atlantic rain forest trees: a comparative study.

Biotropica, v.32, n.4b, p.811-823, 2000.

NEWSTROM, L. E. et al. Diversity of long-term flowering patterns. In: MCDADE, L. A. et al. (Ed.). La selva: Ecology and natural history of a neotropical rain forest. Chicago: University Chicago, 1994. p.142-160.

PACHECO, M. V. et al. Physiological quality evaluation of the radii Schinus terebinthifolius seeds. Revista Brasileira de Sementes, v.33, n.4, p.762-767, 2011.

PAISE, G.; VIEIRA, E. M. Produção de frutos e distribuição espacial de angiospermas com frutos zoocóricos em uma Floresta Ombrófila Mista no Rio Grande do Sul, Brasil. Revista Brasileira de Botânica, v.28, n.3, p.615-625, 2005.

PEREIRA, S. R. et al. Tamanho de frutos e de sementes e sua influência na germinação de jatobá-do-cerrado (Hymenaea stigonocarpa var. stigonocarpa Mart. ex Hayne, Leguminosae Caesalpinoideae). Revista Brasileira de Sementes, v.33, n.1, p.141-148, 2011.
RODRIGUES, R. R.; BRANCALION, P. H. S.; ISERnhagen, I. (Org.) Pacto para a restauração ecológica da Mata Atlântica: referencial dos conceitos e ações de restauração florestal. São Paulo: Instituto BioAtlântica, 2009. 256p.

SERGIPE. Secretaria de Estado do Planejamento e da Ciência e Tecnologia- SEPLANTEC.

Superintendência de Estudos e Pesquisas-SUPES. Informes Municipais. Aracaju: 2000. 75p.

STEPHENSON, A. G. Flower and fruit abortion: proximate causes and ultimate functions.

Annual Review of Ecology and Systematics, v.12, p.253-279, 1981.

VENCOVSKY, R. Herança quantitativa. In: PATERNIANI, E.; VIEGAS, G. P.

Melhoramento e produção do milho. 2.ed. Campinas: Fundação Cargill, 1987. p.137-214.

VIEIRA, F. A.; CARVALHO, D. Maturação e morfometria dos frutos de Miconia albicans (Swartz) Triana (Melastomataceae) em um remanescente de floresta estacional semidecídua montana em Lavras, MG. Revista Árvore, v.33, n.6, p.1015-1023, 2009.

WIELEWICKI, A. P. et al. Proposta de padrões de germinação e teor de água para sementes de algumas espécies florestais presentes na região sul do Brasil. Revista Brasileira de Sementes, v.28, n.3, p.191-197, 2006. 